\title{
$H$-FUNCTION WITH COMPLEX PARAMETERS I: EXISTENCE
}

\author{
FADHEL A. AL-MUSALLAM and VU KIM TUAN
}

(Received 10 November 1998 and in revised form 17 December 1999)

\begin{abstract}
An $H$-function with complex parameters is defined by a Mellin-Barnes type integral. Necessary and sufficient conditions under which the integral defining the $H$-function converges absolutely are established. Some properties, special cases, and an application to integral transforms are given.
\end{abstract}

2000 Mathematics Subject Classification. Primary 33C60.

\section{Introduction. Let}

$$
k(s)=\frac{\prod_{j=1}^{P} \Gamma\left(p_{j}+\alpha_{j} s\right)}{\prod_{j=1}^{Q} \Gamma\left(q_{j}+\beta_{j} s\right)} .
$$

Here, $P$ and $Q$ are nonnegative integers, $p_{j}(1 \leq j \leq P), q_{j}(1 \leq j \leq Q)$ are complex numbers, and $\alpha_{j}(1 \leq j \leq P), \beta_{j}(1 \leq j \leq Q)$ are nonzero complex numbers. Throughout the paper, an empty product is interpreted as unity and an empty sum as zero.

Let $c$ be a real number such that

$$
c \neq \operatorname{Re}\left(-\frac{p_{j}}{\alpha_{j}}\right), \quad \forall j \text { with } \operatorname{Re} \alpha_{j}=0,
$$

and let $\Lambda_{c}$ denote a contour from $c-i \infty$ to $c+i \infty$ not passing through any pole of $k(s)$ and satisfies the following.

(1) If $\operatorname{Re} \alpha_{j}>0$, then all the points

$$
s=-\frac{m}{\alpha_{j}}-\frac{p_{j}}{\alpha_{j}}, \quad m=0,1,2, \ldots
$$

are to the left of $\Lambda_{c}$.

(2) If $\operatorname{Re} \alpha_{j}<0$ then all the points (1.3) are to the right of $\Lambda_{c}$.

(3) If $\operatorname{Re} \alpha_{j}=0$ and $\operatorname{Re}\left(-p_{j} / \alpha_{j}\right)<c$, then all the points (1.3) are to the left of $\Lambda_{c}$.

(4) If $\operatorname{Re} \alpha_{j}=0$, and $\operatorname{Re}\left(-p_{j} / \alpha_{j}\right)>c$, then all the points (1.3) are to the right of $\Lambda_{c}$.

We remark that such a contour $\Lambda_{c}$ always exists. Geometrically, the poles of each gamma function in the numerator of $k(s)$ lie on a half-line, and the conditions on $\Lambda_{c}$ ensure that the contour does not cut any of these half-lines.

We define the $H$-function with complex parameters as a Mellin-Barnes type integral, (see [2]), as

$$
H_{Q}^{P}\left(\begin{array}{l|l}
z, c \mid & \begin{array}{c}
(p, \alpha)_{P} \\
(q, \beta)_{Q}
\end{array}
\end{array}\right)=\frac{1}{2 \pi i} \int_{\Lambda_{c}} k(s) z^{-s} d s,
$$


where $k(s)$ is as in (1.1). Here, and elsewhere in this paper, $(p, \alpha)_{P}=\left(p_{1}, \alpha_{1}\right)$, $\left(p_{2}, \alpha_{2}\right), \ldots,\left(p_{P}, \alpha_{P}\right)$, and similarly for $(q, \beta)_{Q}$. The conditions put on $\Lambda_{c}$ ensure that the $H$-function, if it exists, is independent of the contour $\Lambda_{c}$.

The integral in (1.4) with complex $p_{j}(1 \leq j \leq P), q_{j}(1 \leq j \leq Q)$, but the parameters $\alpha_{j}(1 \leq j \leq P)$ and $\beta_{j}(1 \leq j \leq Q)$ are all real, was introduced in [13] and its theory was developed in $[2,12]$. The conditions for its existence which are indicated in [4, Section 1.19] were first proved in [3]. Different conditions for the existence of the integral in (1.4), with real $\alpha_{j}(1 \leq j \leq P)$ and $\beta_{j}(1 \leq j \leq Q)$, for two contours other than $\Lambda_{c}$, were given in [14, Section 8.3.1] and [15].

In a series of papers, Meijer [11] studied the properties of the function defined by (1.4) when $\alpha_{j}= \pm 1,(1 \leq j \leq P)$, and $\beta_{j}= \pm 1,(1 \leq j \leq Q)$. This function turned out to be a generalization of the generalized hypergeometric function ${ }_{p} F_{q}(z)$ (see $[1,4])$ and is called the Meijer $G$-function. In 1961, Fox [5] considered the integral (1.4) for real $\alpha_{j}(1 \leq j \leq P)$ and $\beta_{j}(1 \leq j \leq Q)$ as an example of a symmetric Fourier kernel. Subsequently, when $\alpha_{j}$ and $\beta_{j}$ are restricted to be real numbers, the function defined by (1.4) was called the Fox $H$-function. It includes not only the $G$-function, but also functions such as the Mittag-Leffler function. The Meijer $G$-function and Fox $H$-function play an important role in applications and many works have been devoted to the study of those functions $[4,7,8,9,10,14,15,16]$.

The $H$-function (1.4), where $\alpha_{j}(1 \leq j \leq P)$ and $\beta_{j}(1 \leq j \leq Q)$ are complex numbers, is not considered in the literature. In this case the gamma functions in the numerator of (1.4) have poles in many directions that are not necessarily horizontal. This case is interesting and important since many functions can be expressed as $H$-functions with complex parameters but not as $H$-functions in the classical sense.

We determine necessary and sufficient conditions (Theorem 3.2), intrinsically related to the parameters, under which the integral defining the $H$-function converges absolutely. The conditions we derive, naturally, reduce to those of the classical case where the $\alpha_{j}$ 's and $\beta_{j}$ 's are real [4]. It is striking that the integral in (1.4) converges only if $\sum_{j=1}^{P} \operatorname{Im} \alpha_{j}-\sum_{j=1}^{Q} \operatorname{Im} \beta_{j}=0$, or roughly speaking, if $(\alpha)_{P}$ and $(\beta)_{Q}$ are almost "real" vectors. The integral may, in general, converge conditionally under weaker conditions. As examples of the $H$-function with complex parameters we list, in Section 5, some well-known integrals that are not special cases of the classical Fox $H$-function. In Section 6, we consider the $H$-function with complex parameters as a Fourier kernel of some integral transform.

In what follows, we determine sufficient conditions that enable us to compute the $H$-function as a sum of residues at the right or the left poles of $k(s)$. There we compute the $H$-function in particular cases and give applications to integral transforms.

2. Asymptotic expansion of the integrand $k(s)$. We write $f(z) \simeq g(z)$, as $|z| \rightarrow \infty$, $\alpha \leq \arg z \leq \beta$, if $f(z)=g(z)[1+o(1)]$, as $|z| \rightarrow \infty$, within the sector $\alpha \leq \arg z \leq \beta$.

On the other hand, we write $f(z) \asymp g(z)$, as $|z| \rightarrow \infty$, within the sector $\alpha \leq \arg z \leq \beta$, if there are positive constants $W_{1}$ and $W_{2}$ such that

$$
W_{2}|g(z)| \leq|f(z)| \leq W_{1}|g(z)|, \quad \text { as }|z| \rightarrow \infty, \alpha \leq \arg z \leq \beta \text {. }
$$


In what follows, we use $\operatorname{Arg} w$ to denote the principal argument of $w$, that is, $-\pi<$ $\operatorname{Arg} w \leq \pi$, and adapt the convention that $\operatorname{Arg}(0)=0$.

LEMMA 2.1. If $p$ and $\alpha \neq 0$ are complex numbers, then for $|s| \rightarrow \infty$, Re $s \geq 0$,

$$
\begin{aligned}
|\Gamma(p+\alpha s)| \simeq & (2 \pi)^{1 / 2}|\alpha|^{-(1 / 2)+\operatorname{Re} p} e^{-[\operatorname{Arg}(\alpha S(\alpha, s))+\operatorname{Arg} s] \operatorname{Im} p} \\
& \times|s|^{-(1 / 2)+\operatorname{Re} p} e^{(\ln |s|)(\operatorname{Re} s) \operatorname{Re} \alpha} e^{-(\ln |s|)(\operatorname{Im} s) \operatorname{Im} \alpha} \\
& \times e^{-(\operatorname{Re} s)\{[\operatorname{Arg}(\alpha S(\alpha, s))+\operatorname{Arg} s] \operatorname{Im} \alpha-(\ln |\alpha|-1) \operatorname{Re} \alpha\}} \\
& \times e^{-(\operatorname{Im} s)\{[\operatorname{Arg}(\alpha S(\alpha, s))+\operatorname{Arg} s] \operatorname{Re} \alpha+(\ln |\alpha|-1) \operatorname{Im} \alpha\}} \\
& \times\left|\frac{1}{2}(1+S(\alpha, s))+(1-S(\alpha, s)) \sin \pi(p+\alpha s)\right|^{-1}
\end{aligned}
$$

where

$$
\begin{aligned}
S(\alpha, s) & =\operatorname{signRe} \alpha+[|\operatorname{signRe} \alpha|-1](\operatorname{signIm} \alpha) \operatorname{signIm} s \\
& = \begin{cases}-(\operatorname{signIm} \alpha) \operatorname{signIm} s, & \operatorname{Re} \alpha=0 \\
\operatorname{signRe} \alpha, & \operatorname{Re} \alpha \neq 0\end{cases}
\end{aligned}
$$

Proof. We use the asymptotic expansion of the gamma function $\Gamma(a+s)$ for $|s| \rightarrow \infty$ and $a$ being bounded, (see [4]),

$$
\Gamma(a+s) \simeq(2 \pi)^{1 / 2} s^{s+a-(1 / 2)} e^{-s}, \quad|\operatorname{Arg}(a+s)| \leq \pi-\varepsilon, \quad \varepsilon>0 .
$$

Observe that if $z \neq 0$ and $w$ are complex numbers, then

$$
\left|z^{w} e^{-z}\right|=|z|^{\operatorname{Re} w} e^{-(\operatorname{Arg} z) \operatorname{Im} w-\operatorname{Re} z}
$$

Suppose that $\operatorname{Re} s \geq 0$, and $|\operatorname{Arg} \alpha|<(\pi / 2)(\operatorname{Re} \alpha>0)$. Then $\operatorname{Arg}(\alpha s)=\operatorname{Arg} \alpha+\operatorname{Arg} s$, $|\operatorname{Arg}(p+\alpha s)| \leq \pi-\varepsilon, \varepsilon>0$, and (2.4) along with (2.5) give

$$
\begin{aligned}
|\Gamma(p+\alpha s)| \simeq & (2 \pi)^{1 / 2}\left[(|\alpha||s|)^{\operatorname{Re}(\alpha s-(1 / 2)+p)} e^{-(\operatorname{Arg}(\alpha s)) \operatorname{Im}(\alpha s-(1 / 2)+p)}\right] \\
& \times e^{-(\operatorname{Re} \alpha) \operatorname{Re} s+(\operatorname{Im} \alpha) \operatorname{Im} s} .
\end{aligned}
$$

A computation shows that

$$
\begin{aligned}
|\Gamma(p+\alpha s)| \simeq & (2 \pi)^{1 / 2}|\alpha|^{-(1 / 2)+\operatorname{Re} p} e^{-(\operatorname{Arg} \alpha+\operatorname{Arg} s) \operatorname{Im} p}|s|^{-(1 / 2)+\operatorname{Re} p} \\
& \times e^{(\ln |s|)(\operatorname{Re} s) \operatorname{Re} \alpha-(\ln |s|)(\operatorname{Im} s) \operatorname{Im} \alpha} \\
& \times e^{-(\operatorname{Re} s)\{[\operatorname{Arg} \alpha+\operatorname{Arg} s] \operatorname{Im} \alpha-(\ln |\alpha|-1) \operatorname{Re} \alpha\}} \\
& \times e^{-\operatorname{Im}(s)\{[\operatorname{Arg} \alpha+\operatorname{Arg} s] \operatorname{Re} \alpha+(\ln |\alpha|-1) \operatorname{Im} \alpha\}} .
\end{aligned}
$$

If $\operatorname{Re} s \geq 0$ and $\operatorname{Re} \alpha<0$, then replacing $\alpha$ and $p$ in (2.7) by $-\alpha$ and $1-p$, respectively, we obtain the asymptotic expansion of $\Gamma(1-p-\alpha s)$, and since by the reflection formula 
of the gamma function, (see [4]),

$$
\Gamma(p+\alpha s)=\frac{\pi}{\Gamma(1-p-\alpha s) \sin \pi(p+\alpha s)},
$$

we obtain for $\operatorname{Re} s \geq 0$ and $\operatorname{Re} \alpha<0$,

$$
\begin{aligned}
|\Gamma(p+\alpha s)| \simeq & (2 \pi)^{1 / 2}|\alpha|^{-(1 / 2)+\operatorname{Re} p} e^{-[\operatorname{Arg}(-\alpha)+\operatorname{Arg} s] \operatorname{Im} p}|s|^{-(1 / 2)+\operatorname{Re} p} \\
& \times e^{(\ln |s|)(\operatorname{Re} s) \operatorname{Re} \alpha} e^{-(\ln |s|)(\operatorname{Im} s) \operatorname{Im} \alpha} \\
& \times e^{-(\operatorname{Re} s)\{[\operatorname{Arg}(-\alpha)+\operatorname{Arg} s] \operatorname{Im} \alpha-(\ln |\alpha|-1) \operatorname{Re} \alpha\}} \\
& \times e^{-\operatorname{Im}(s)\{[\operatorname{Arg}(-\alpha)+\operatorname{Arg} s] \operatorname{Re} \alpha+(\ln |\alpha|-1) \operatorname{Im} \alpha\}} \\
& \times|2 \sin \pi(p+\alpha s)|^{-1}
\end{aligned}
$$

Now, suppose that $\operatorname{Re} \alpha=0$ and $\operatorname{Im} \alpha>0$. To study the asymptotic behaviour of $|\Gamma(p+\alpha s)|$, as $|s| \rightarrow \infty$, in the half-plane Re $s \geq 0$, we consider separately this behaviour for $s$ in the fourth and first quadrants.

(i) $\operatorname{Im} s<0$ ( $s$ is in the fourth quadrant).

In this case $\operatorname{Arg}(p+\alpha s) \leq \pi-\varepsilon$, and the expansion is

$$
\begin{aligned}
|\Gamma(p+\alpha s)| \simeq & (2 \pi)^{1 / 2}\left[(|\alpha||s|)^{\operatorname{Re}(\alpha s-(1 / 2)+p)} e^{-\operatorname{Arg}(\alpha s) \operatorname{Im}(\alpha s-(1 / 2)+p)}\right] e^{-(\operatorname{Re} \alpha) \operatorname{Re} s+(\operatorname{Im} \alpha) \operatorname{Im} s} \\
= & (2 \pi)^{1 / 2}|\alpha|^{-(1 / 2)+\operatorname{Re} p} e^{-[\operatorname{Arg} \alpha+\operatorname{Arg} s] \operatorname{Im} p}|s|^{-(1 / 2)+\operatorname{Re} p} \\
& \times e^{-(\ln |s|)(\operatorname{Im} s) \operatorname{Im} \alpha} e^{-(\operatorname{Re} s)[\operatorname{Arg} \alpha+\operatorname{Arg} s] \operatorname{Im} \alpha} e^{-(\operatorname{Im} s)(\ln |\alpha|-1) \operatorname{Im} \alpha} .
\end{aligned}
$$

(ii) $\operatorname{Im} s \geq 0$ ( $s$ is in the first quadrant).

In this case the expansion is

$$
\begin{aligned}
|\Gamma(p+\alpha s)| \simeq & (2 \pi)^{1 / 2}|\alpha|^{-(1 / 2)+\operatorname{Re} p} e^{-[\operatorname{Arg}(-\alpha)+\operatorname{Arg} s] \operatorname{Im} p}|s|^{-(1 / 2)+\operatorname{Re} p} \\
& \times e^{-(\ln |s|)(\operatorname{Im} s) \operatorname{Im} \alpha} e^{-(\operatorname{Re} s)[\operatorname{Arg}(-\alpha)+\operatorname{Arg} s] \operatorname{Im} \alpha} e^{-(\operatorname{Im} s)(\ln |\alpha|-1) \operatorname{Im} \alpha} \\
& \times|2 \sin \pi(p+\alpha s)|^{-1} .
\end{aligned}
$$

Combining (2.10) and (2.11) we obtain for $\operatorname{Re} \alpha=0$ and $\operatorname{Im} \alpha>0$;

$$
\begin{aligned}
|\Gamma(p+\alpha s)| \simeq & (2 \pi)^{1 / 2}|\alpha|^{-(1 / 2)+\operatorname{Re} p} e^{-[\operatorname{Arg}(-\alpha \operatorname{signIm} s)+\operatorname{Arg} s] \operatorname{Im} p}|s|^{-(1 / 2)+\operatorname{Re} p} \\
& \times e^{-(\ln |s|)(\operatorname{Im} s) \operatorname{Im} \alpha} e^{-(\operatorname{Re} s)[\operatorname{Arg}(-\alpha \operatorname{signIm} s)+\operatorname{Arg} s] \operatorname{Im} \alpha} e^{-(\operatorname{Im} s)(\ln |\alpha|-1) \operatorname{Im} \alpha} \\
& \times\left|\frac{1}{2}(1-\operatorname{signIm} s)+(1+\operatorname{signIm} s) \sin \pi(p+\alpha s)\right|^{-1} .
\end{aligned}
$$

If $\operatorname{Re} s \geq 0, \operatorname{Re} \alpha=0$, and $\operatorname{Im} \alpha<0$, then (2.12) with $p$ and $\alpha$ replaced by $1-p$ and $-\alpha$, respectively, yields the asymptotic expansion of $\Gamma(1-p-\alpha s)$, and with the aid of the reflection formula (2.8) we obtain for $\operatorname{Re} \alpha=0$ and $\operatorname{Im} \alpha<0$,

$$
\begin{aligned}
|\Gamma(p+\alpha s)| \simeq & (2 \pi)^{1 / 2}|\alpha|^{-(1 / 2)+\operatorname{Re} p} e^{-[\operatorname{Arg}(\alpha \operatorname{signIm} s)+\operatorname{Arg} s] \operatorname{Im} p}|s|^{-(1 / 2)+\operatorname{Re} p} \\
& \times e^{-(\ln |s|)(\operatorname{Im} s) \operatorname{Im} \alpha} e^{-(\operatorname{Re} s)[\operatorname{Arg}(\alpha \operatorname{signIm} s)+\operatorname{Arg} s] \operatorname{Im} \alpha} e^{-(\operatorname{Im} s)(\ln |\alpha|-1) \operatorname{Im} \alpha} \\
& \times\left.\left|\frac{1}{2}(1+\operatorname{signIm} s)+(1-\operatorname{signIm} s)\right| \sin \pi(p+\alpha s)\right|^{-1} .
\end{aligned}
$$

Combining (2.7), (2.9), (2.12), and (2.13) we obtain (2.2). 
Applying (2.2) gives, as $|s| \rightarrow \infty$ and $\operatorname{Re} s \geq 0$,

$$
\begin{aligned}
\left|\prod_{j=1}^{P} \Gamma\left(p_{j}+\alpha_{j} s\right)\right| \simeq & (2 \pi)^{P / 2} \prod_{j=1}^{P}\left|\alpha_{j}\right|^{-(1 / 2)+\operatorname{Re} p_{j}} \\
& \times e^{-\sum_{j=1}^{P}\left[\operatorname{Arg}\left(\alpha_{j} S\left(\alpha_{j}, s\right)\right)+\operatorname{Arg} s\right] \operatorname{Im} p_{j}}|s|^{-(P / 2)+\sum_{j=1}^{P} \operatorname{Re} p_{j}} \\
& \times e^{(\ln |s|)(\operatorname{Re} s) \sum_{j=1}^{P} \operatorname{Re} \alpha_{j}} e^{-(\ln |s|)(\operatorname{Im} s) \sum_{j=1}^{P} \operatorname{Im} \alpha_{j}} \\
& \times e^{-(\operatorname{Re} s) \sum_{j=1}^{P}\left\{\left[\operatorname{Arg}\left(\alpha_{j} S\left(\alpha_{j}, s\right)\right)+\operatorname{Arg} s\right] \operatorname{Im} \alpha_{j}-\left(\ln \left|\alpha_{j}\right|-1\right) \operatorname{Re} \alpha_{j}\right\}} \\
& \times e^{-(\operatorname{Im} s) \sum_{j=1}^{P}\left\{\left[\operatorname{Arg}\left(\alpha_{j} S\left(\alpha_{j}, s\right)\right)+\operatorname{Arg} s\right] \operatorname{Re} \alpha_{j}+\left(\ln \left|\alpha_{j}\right|-1\right) \operatorname{Im} \alpha_{j}\right\}} \\
& \times \prod_{j=1}^{P}\left|\frac{1}{2}\left(1+S\left(\alpha_{j}, s\right)\right)+\left(1-S\left(\alpha_{j}, s\right)\right) \sin \pi\left(p_{j}+\alpha_{j} s\right)\right|^{-1} .
\end{aligned}
$$

We obtain a similar expansion for $\left|\prod_{j=1}^{Q} \Gamma\left(q_{j}+\beta_{j} s\right)\right|$, as $|s| \rightarrow \infty$ and $\operatorname{Re} s \geq 0$.

Now, we are ready to obtain the asymptotic expansion of $|k(s)|$, but in order to simplify the expression we first observe that

$$
\operatorname{Arg}(\alpha S(\alpha, s))= \begin{cases}-\frac{\pi}{2} \operatorname{signIm} s, & \operatorname{Re} \alpha=0, \\ \operatorname{Arg}(\alpha \operatorname{signRe} \alpha), & \operatorname{Re} \alpha \neq 0,\end{cases}
$$

so

$$
\begin{aligned}
\sum_{j=1}^{P}\{[ & \left.\left.\operatorname{Arg}\left(\alpha_{j} S\left(\alpha_{j}, s\right)\right)+\operatorname{Arg} s\right] \operatorname{Im} \alpha_{j}-\left(\ln \left|\alpha_{j}\right|-1\right) \operatorname{Re} \alpha_{j}\right\} \\
= & -\frac{\pi}{2}(\operatorname{signIm} s) \sum_{\operatorname{Re} \alpha_{j}=0} \operatorname{Im} \alpha_{j}+\left(\sum_{j=1}^{P} \operatorname{Im} \alpha_{j}\right) \operatorname{Arg} s \\
& +\sum_{\operatorname{Re} \alpha_{j} \neq 0}\left[\operatorname{Arg}\left(\alpha_{j} \operatorname{sign\operatorname {Re}} \alpha_{j}\right) \operatorname{Im} \alpha_{j}-\left(\ln \left|\alpha_{j}\right|-1\right) \operatorname{Re} \alpha_{j}\right]
\end{aligned}
$$

Since $\operatorname{Arg}(-\alpha)=\operatorname{Arg} \alpha-\pi \operatorname{signIm} \alpha$, provided that $\operatorname{Im} \alpha \neq 0$; we see that $\operatorname{Arg}(-\alpha)$ $\operatorname{Im} \alpha=(\operatorname{Arg} \alpha) \operatorname{Im} \alpha-\pi|\operatorname{Im} \alpha|$, for all $\alpha$. Moreover, $(\operatorname{Arg} \alpha) \operatorname{Im} \alpha=(\pi / 2)|\operatorname{Im} \alpha|$, whenever $\operatorname{Re} \alpha=0$. Thus, a computation shows that

$$
\begin{aligned}
& \sum_{\operatorname{Re} \alpha_{j} \neq 0}\left[\operatorname{Arg}\left(\alpha_{j} \operatorname{signRe} \alpha_{j}\right) \operatorname{Im} \alpha_{j}-\left(\ln \left|\alpha_{j}\right|-1\right) \operatorname{Re} \alpha_{j}\right] \\
& =-\frac{\pi}{2} \sum_{\operatorname{Re} \alpha_{j}=0}\left|\operatorname{Im} \alpha_{j}\right|+\sum_{j=1}^{P} \operatorname{Re} \alpha_{j}+\sum_{\operatorname{Re} \alpha_{j} \geq 0}\left[\left(\operatorname{Arg} \alpha_{j}\right) \operatorname{Im} \alpha_{j}-\left(\ln \left|\alpha_{j}\right|\right) \operatorname{Re} \alpha_{j}\right] \\
& \quad+\sum_{\operatorname{Re} \alpha_{j}<0}\left[\operatorname{Arg}\left(-\alpha_{j}\right) \operatorname{Im} \alpha_{j}-\left(\ln \left|\alpha_{j}\right|\right) \operatorname{Re} \alpha_{j}\right] \\
& =\sum_{j=1}^{P}\left[\left(\operatorname{Arg} \alpha_{j}\right) \operatorname{Im} \alpha_{j}-\left(\ln \left|\alpha_{j}\right|\right) \operatorname{Re} \alpha_{j}\right]-\pi \sum_{\operatorname{Re} \alpha_{j}<0}\left|\operatorname{Im} \alpha_{j}\right|-\frac{\pi}{2} \sum_{\operatorname{Re} \alpha_{j}=0}\left|\operatorname{Im} \alpha_{j}\right|+\sum_{j=1}^{P} \operatorname{Re} \alpha_{j} .
\end{aligned}
$$


It is clear that $\operatorname{Arg}(\alpha S(\alpha, s) \operatorname{Re} \alpha)=\operatorname{Arg}(\alpha \operatorname{signRe} \alpha) \operatorname{Re} \alpha$, for all $\alpha$, and therefore

$$
\begin{aligned}
& \sum_{j=1}^{P}\left\{\left[\operatorname{Arg}\left(\alpha_{j} S\left(\alpha_{j}, s\right)\right)+\operatorname{Arg} s\right] \operatorname{Re} \alpha_{j}+\left(\ln \left|\alpha_{j}\right|-1\right) \operatorname{Im} \alpha_{j}\right\} \\
& \quad=\sum_{j=1}^{P}\left[\operatorname{Arg}\left(\alpha_{j} \operatorname{sign}\left(\operatorname{Re} \alpha_{j}\right)\right) \operatorname{Re} \alpha_{j}+\left(\ln \left|\alpha_{j}\right|\right) \operatorname{Im} \alpha_{j}\right]-\sum_{j=1}^{P} \operatorname{Im} \alpha_{j}+\left(\sum_{j=1}^{P} \operatorname{Re} \alpha_{j}\right) \operatorname{Arg} s .
\end{aligned}
$$

Let

$$
\begin{aligned}
\Delta_{0}= & -\frac{1}{2}(P-Q)+\sum_{j=1}^{P} \operatorname{Re} p_{j}-\sum_{j=1}^{Q} \operatorname{Re} q_{j}, \\
L= & \sum_{j=1}^{P}\left[\left(\operatorname{Arg} \alpha_{j}\right) \operatorname{Im} \alpha_{j}-\left(\ln \left|\alpha_{j}\right|\right) \operatorname{Re} \alpha_{j}\right]-\sum_{j=1}^{Q}\left[\left(\operatorname{Arg} \beta_{j}\right) \operatorname{Im} \beta_{j}-\left(\ln \left|\beta_{j}\right|\right) \operatorname{Re} \beta_{j}\right] \\
M= & \sum_{j=1}^{P}\left[\operatorname{Arg}\left(\alpha_{j} \operatorname{signRe} \alpha_{j}\right) \operatorname{Re} \alpha_{j}+\left(\ln \left|\alpha_{j}\right|\right) \operatorname{Im} \alpha_{j}\right] \\
& -\sum_{j=1}^{Q}\left[\operatorname{Arg}\left(\beta_{j} \operatorname{signRe} \beta_{j}\right) \operatorname{Re} \beta_{j}+\left(\ln \left|\beta_{j}\right|\right) \operatorname{Im} \beta_{j}\right] \\
X= & \pi\left(\sum_{\operatorname{Re} \alpha_{j}<0}\left|\operatorname{Im} \alpha_{j}\right|-\sum_{\operatorname{Re} \beta_{j}<0}\left|\operatorname{Im} \beta_{j}\right|\right)+\frac{\pi}{2}\left(\sum_{\operatorname{Re} \alpha_{j}=0}\left|\operatorname{Im} \alpha_{j}\right|-\sum_{\operatorname{Re} \beta_{j}=0}\left|\operatorname{Im} \beta_{j}\right|\right) \\
\mu & \sum_{j=1}^{P} \operatorname{Im} \alpha_{j}-\sum_{j=1}^{Q} \operatorname{Im} \beta_{j}, \quad T=\sum_{j=1}^{P} \operatorname{Re} \alpha_{j}-\sum_{j=1}^{Q} \operatorname{Re} \beta_{j} .
\end{aligned}
$$

Define

$$
\begin{aligned}
m(s)= & \sum_{j=1}^{P}\left[\operatorname{Arg}\left(\alpha_{j} S\left(\alpha_{j}, s\right)\right)+\operatorname{Arg} s\right] \operatorname{Im} p_{j}-\sum_{j=1}^{Q}\left[\operatorname{Arg}\left(\beta_{j} S\left(\beta_{j}, s\right)\right)+\operatorname{Arg} s\right] \operatorname{Im} q_{j} \\
= & -\frac{\pi}{2}(\operatorname{signIm} s)\left(\sum_{\operatorname{Re} \alpha_{j}=0} \operatorname{Im} p_{j}-\sum_{\operatorname{Re} \beta_{j}=0} \operatorname{Im} q_{j}\right)+\left(\sum_{j=1}^{P} \operatorname{Im} p_{j}-\sum_{j=1}^{Q} \operatorname{Im} q_{j}\right) \operatorname{Arg} s \\
& +\sum_{\operatorname{Re} \alpha_{j} \neq 0} \operatorname{Arg}\left(\alpha_{j} \operatorname{signRe} \alpha_{j}\right) \operatorname{Im} p_{j}-\sum_{\operatorname{Re} \beta_{j} \neq 0} \operatorname{Arg}\left(\beta_{j} \operatorname{signRe} \beta_{j}\right) \operatorname{Im} q_{j},
\end{aligned}
$$

and finally, let

$$
Y(s)=(2 \pi)^{(P-Q) / 2} \frac{\prod_{j=1}^{P}\left|\alpha_{j}\right|^{-(1 / 2)+\operatorname{Re} p_{j}}}{\prod_{j=1}^{Q}\left|\beta_{j}\right|^{-(1 / 2)+\operatorname{Re} q_{j}}} e^{-m(s)} .
$$

With the above notation in mind, we get from (2.14), and a similar expansion for $\left|\prod_{j=1}^{Q} \Gamma\left(q_{j}+\beta_{j} s\right)\right|$, the asymptotic expansion of $|k(s)|$, as $|s| \rightarrow \infty$ and Re $s \geq 0$. We state this in the following lemma. 
LEMMA 2.2. We have

$$
\begin{aligned}
\left|\frac{\prod_{j=1}^{P} \Gamma\left(p_{j}+\alpha_{j} s\right)}{\prod_{j=1}^{Q} \Gamma\left(q_{j}+\beta_{j} s\right)}\right| \simeq & Y(s)|s|^{\Delta_{0}} e^{T(\ln |s|) \operatorname{Re} s} e^{-\mu(\ln |s|) \operatorname{Im} s} e^{-(\operatorname{Im} s)(M-\mu+T \operatorname{Arg} s)} \\
& \times e^{-(\operatorname{Re} s)\left[L+T-X+\mu \operatorname{Arg} s-(\pi / 2)(\operatorname{signIm} s)\left(\sum_{\operatorname{Re} \alpha_{j}=0} \operatorname{Im} \alpha_{j}-\sum_{\operatorname{Re} \beta_{j}=0} \operatorname{Im} \beta_{j}\right)\right]} \\
& \times \frac{\prod_{j=1}^{Q}\left|(1 / 2)\left(1+S\left(\beta_{j}, s\right)\right)+\left(1-S\left(\beta_{j}, s\right)\right) \sin \pi\left(q_{j}+\beta_{j} s\right)\right|}{\prod_{j=1}^{P}\left|(1 / 2)\left(1+S\left(\alpha_{j}, s\right)\right)+\left(1-S\left(\alpha_{j}, s\right)\right) \sin \pi\left(p_{j}+\alpha_{j} s\right)\right|}, \quad \operatorname{Re} s \geq 0 .
\end{aligned}
$$

\section{Existence of the $H$-function with complex parameters}

LEMMA 3.1. Let $b_{j}$ be a nonzero real number, $q_{j}$ be a complex number, $j=1,2, \ldots, n$, and

$$
g(t)=\prod_{j=1}^{n}\left|\frac{1}{2}\left(1+S\left(b_{j} i, i t\right)\right)+\left(1-S\left(b_{j} i, i t\right)\right) \sin \pi\left(q_{j}-b_{j} t\right)\right| .
$$

Then, the integral

$$
I=\int_{-\infty}^{\infty} g(t)(|t|+1)^{p} e^{-\alpha t \ln |t|-\beta|t|-\gamma t} d t
$$

converges if and only if

(i) $\alpha=0$ and $|\gamma|<\beta$, or

(ii) $\alpha=0,|\gamma|=\beta$, and $p<-1$.

Proof. Let

$$
\varphi(t)=(p+1) \ln (|t|+1)-\alpha t \ln |t|-\beta|t|-\gamma t, \quad t \neq 0 .
$$

If $\alpha<0$, then $\lim _{t \rightarrow \infty} \varphi(t)=\infty$ and therefore, there is an $X>0$ such that $\varphi(t)>0$, for $t>X$. Hence,

$$
e^{\varphi(t)}=(|t|+1)^{p+1} e^{-\alpha t \ln |t|-\beta|t|-\gamma t}>1 \text { for } t>X .
$$

Since $S\left(b_{j} i, i t\right)=-\left(\operatorname{sign} b_{j}\right) \operatorname{sign} t$, it follows that $I=I_{1}+I_{2}$, where

$$
\begin{aligned}
& I_{1}=\int_{-\infty}^{0}\left(\prod_{b_{j}<0}\left|2 \sin \pi\left(q_{j}-b_{j} t\right)\right|\right)(|t|+1)^{p} e^{-\alpha t \ln |t|-\beta|t|-\gamma t} d t, \\
& I_{2}=\int_{0}^{\infty}\left(\prod_{b_{j}>0}\left|2 \sin \pi\left(q_{j}-b_{j} t\right)\right|\right)(|t|+1)^{p} e^{-\alpha t \ln |t|-\beta|t|-\gamma t} d t .
\end{aligned}
$$

Thus, (3.4) implies that

$$
\begin{aligned}
I & \geq I_{2} \geq \int_{X}^{\infty}\left(\prod_{b_{j}>0}\left|2 \sin \pi\left(q_{j}-b_{j} t\right)\right|\right)(|t|+1)^{p} e^{-\alpha t \ln |t|-\beta|t|-\gamma t} d t \\
& >\int_{X}^{\infty} \frac{1}{|t|+1} \prod_{b_{j}>0}\left|2 \sin \pi\left(q_{j}-b_{j} t\right)\right| d t .
\end{aligned}
$$

Since $\int_{X}^{\infty}(1 /(|t|+1)) \prod_{b_{j}>0}\left|2 \sin \pi\left(q_{j}-b_{j} t\right)\right| d t=\infty$, the integral (3.2) is divergent. 
Similarly, since $\lim _{t \rightarrow-\infty} \varphi(t)=\infty$, if $\alpha>0$ and $I \geq I_{1}$, we see that the integral (3.2) is divergent if $\alpha>0$. We have shown that (3.2) is divergent if $\alpha \neq 0$.

Suppose now that $\alpha=0$ and $\beta<|\gamma|$. If $\gamma \geq 0$, then $\beta-\gamma<0$, and therefore, $\lim _{t \rightarrow-\infty} \varphi(t)=\infty$. If $\gamma<0$, then $\beta+\gamma<0$, and therefore, $\lim _{t \rightarrow \infty} \varphi(t)=\infty$. Hence, as before, the integral (3.2) is divergent.

Suppose that $\alpha=0,|\gamma| \leq \beta$, and $p \geq-1$. In this case,

$$
\begin{aligned}
(|t|+1)^{p} e^{-\alpha t \ln |t|-\beta|t|-\gamma t} & = \begin{cases}(|t|+1)^{p}, & \text { if } \gamma \leq 0, t>0, \\
(|t|+1)^{p}, & \text { if } \gamma>0, t<0,\end{cases} \\
& \geq \begin{cases}(|t|+1)^{-1}, & \text { if } \gamma \leq 0, t>0, \\
(|t|+1)^{-1}, & \text { if } \gamma>0, t<0,\end{cases}
\end{aligned}
$$

and hence, the integral (3.2) is divergent since

$$
\begin{aligned}
& I \geq \int_{0}^{\infty} \frac{1}{|t|+1} \prod_{b_{j}>0}\left|2 \sin \pi\left(q_{j}-b_{j} t\right)\right| d t=\infty, \quad \text { when } \gamma \leq 0, \\
& I \geq \int_{-\infty}^{0} \frac{1}{|t|+1} \prod_{b_{j}<0}\left|2 \sin \pi\left(q_{j}-b_{j} t\right)\right| d t=\infty, \text { when } \gamma>0 .
\end{aligned}
$$

If $\alpha=0$ and $|\gamma|<\beta$, then there is an $A>0$ such that

$$
e^{\beta|t|+\gamma t} \geq e^{(\beta-|\gamma|)|t|}>A(1+|t|)^{p+2}, \quad \forall t .
$$

Since $\left|\sin \pi\left(q_{j}-b_{j} t\right)\right| \leq \sqrt{1+\sinh ^{2} \pi \operatorname{Im} q_{j}}$, then (3.9) shows that

$$
I=I_{1}+I_{2} \leq \frac{C}{A} \int_{-\infty}^{\infty}(1+|t|)^{-2} d t<\infty, \quad \text { where } C=\prod_{j=1}^{n} 2 \sqrt{1+\sinh ^{2} \pi \operatorname{Im} q_{j}}
$$

If $\alpha=0, \beta=|\gamma|$, and $p<-1$, then $\beta|t|+\gamma t=|\gamma||t|+\gamma t \geq 0$ for all $t$ and

$$
I \leq C \int_{-\infty}^{\infty}(|t|+1)^{p} e^{-(|y||t|+\gamma t)} d t \leq C \int_{-\infty}^{\infty}(|t|+1)^{p} d t<\infty .
$$

The proof of Lemma 3.1 is now complete.

THEOREM 3.2. Let $k(s)$ be as in (1.1). Let $c$ be an arbitrary real number satisfying (1.2), and let $\Omega_{c}$ denote a contour from $c-i \infty$ to $c+i \infty$ not passing through any pole of the function $k(s)$. If $M$ and $\mu$ are as defined in (2.19) and (2.20), and if

$$
\begin{aligned}
N & =\sum_{j=1}^{P}\left|\operatorname{Re} \alpha_{j}\right|-\sum_{j=1}^{Q}\left|\operatorname{Re} \beta_{j}\right|, \\
\Delta_{c} & =-\frac{1}{2}(P-Q)+\sum_{j=1}^{P} \operatorname{Re} p_{j}-\sum_{j=1}^{Q} \operatorname{Re} q_{j}+c T=\Delta_{0}+c T,
\end{aligned}
$$

then the integral

$$
\int_{\Omega_{c}} k(s) z^{-s} d s
$$


converges absolutely if and only if

(1) $\mu=0, N>0$, and for $|\arg z-M|<(\pi / 2) N$, or

(2) $\mu=0, N \geq 0, \arg z=M \pm(\pi / 2) N$, and $\Delta_{c}=\Delta_{0}+c T<-1$.

Proof. Making the change of variable $s \rightarrow s-c$ we see that

$$
\int_{\Omega_{c}} k(s) z^{-s} d s=z^{-c} \int_{\Omega_{0}} k_{c}(s) z^{-s} d s, \quad k_{c}(s)=\frac{\prod_{j=1}^{P} \Gamma\left(p_{j}+c \alpha_{j}+\alpha_{j} s\right)}{\prod_{j=1}^{Q} \Gamma\left(q_{j}+c \beta_{j}+\beta_{j} s\right)},
$$

where the path of integration $\Omega_{0}$ is the horizontal shifting of the contour $\Omega_{c}$ by $c$ units. Hence, $\int_{\Omega_{c}} k(s) z^{-s} d s$ converges absolutely if and only if $\int_{\Omega_{0}} k_{c}(s) z^{-s} d s$ converges absolutely. Observe that the asymptotic expansion of $k_{c}(s)$ is given by Lemma 2.2 where only $\Delta_{0}$ is replaced by $\Delta_{c}$, and the parameters $p_{j}$ and $q_{j}$ are replaced by $p_{j}+c \alpha_{j}$ and $q_{j}+c \beta_{j}$, respectively, while all other constants involved in the expansion remain unchanged. Thus, without loss of generality, we may assume that $c=0$. Furthermore, because the path of integration coincides with the imaginary axis at infinity, we assume for the rest of the proof that $s=i t, t \in \mathbb{R}$, so $\operatorname{Re} s=0$. Since

$$
\begin{aligned}
& \frac{\prod_{\operatorname{Re} \beta_{j}<0}\left|2 \sin \pi\left(q_{j}+\beta_{j} s\right)\right|}{\prod_{\operatorname{Re} \alpha_{j}<0}\left|2 \sin \pi\left(p_{j}+\alpha_{j} s\right)\right|} \simeq \frac{e^{\pi \sum_{\operatorname{Re} \beta_{j}<0}\left|\operatorname{Im} q_{j}+t \operatorname{Re} \beta_{j}\right|}}{e^{\pi \sum \operatorname{Re} \alpha_{j}<0}\left|\operatorname{Im} p_{j}+t \operatorname{Re} \alpha_{j}\right|},
\end{aligned}
$$

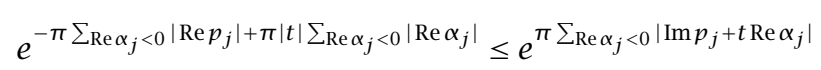

$$
\begin{aligned}
& \leq e^{\pi \sum_{\operatorname{Re} \alpha_{j}<0}\left|\operatorname{Im} p_{j}\right|+\pi|t| \sum_{\operatorname{Re} \alpha_{j}<0}\left|\operatorname{Re} \alpha_{j}\right|},
\end{aligned}
$$

and similarly

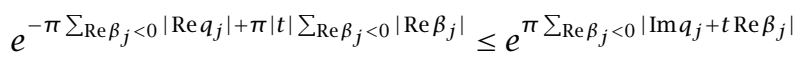

$$
\begin{aligned}
& \leq e^{\pi \sum_{\operatorname{Re} \beta_{j}<0}\left|\operatorname{Im} q_{j}\right|+\pi|t| \sum_{\operatorname{Re} \beta_{j}<0}\left|\operatorname{Re} \beta_{j}\right|},
\end{aligned}
$$

it follows that

$$
\frac{\prod_{\operatorname{Re} \beta_{j}<0}\left|2 \sin \pi\left(q_{j}+\beta_{j} s\right)\right|}{\prod_{\operatorname{Re} \alpha_{j}<0}\left|2 \sin \pi\left(p_{j}+\alpha_{j} s\right)\right|} \asymp e^{\pi|t|\left(\sum_{\operatorname{Re} \alpha_{j}<0}\left|\operatorname{Re} \beta_{j}\right|-\sum_{\operatorname{Re} \beta_{j}<0}\left|\operatorname{Re} \alpha_{j}\right|\right)} .
$$

Now, we deal with the term

$$
\frac{\prod_{\operatorname{Re} \beta_{j}=0}\left|(1 / 2)\left(1+S\left(\beta_{j}, s\right)\right)+\left(1-S\left(\beta_{j}, s\right)\right) \sin \pi\left(q_{j}+\beta_{j} s\right)\right|}{\prod_{\operatorname{Re} \alpha_{j}=0}\left|(1 / 2)\left(1+S\left(\alpha_{j}, s\right)\right)+\left(1-S\left(\alpha_{j}, s\right)\right) \sin \pi\left(p_{j}+\alpha_{j} s\right)\right|} .
$$

Since the path of integration coincides with the imaginary axis at infinity and does not pass through any singular point of the integrand, it follows that $\operatorname{Im}\left(p_{j}\right) \neq 0$ for 
all $j$ such that $\operatorname{Re} \alpha_{j}=0$. Observe that

$$
\begin{aligned}
\min \left\{1,2\left|\sin \pi\left(p_{j}+\alpha_{j} s\right)\right|\right\} & \leq\left|\frac{1}{2}\left(1+S\left(\alpha_{j}, s\right)\right)+\left(1-S\left(\alpha_{j}, s\right)\right) \sin \pi\left(p_{j}+\alpha_{j} s\right)\right| \\
& \leq \max \left\{1,2\left|\sin \pi\left(p_{j}+\alpha_{j} s\right)\right|\right\},
\end{aligned}
$$

and since

$$
0<\left|\sinh \left(\pi \operatorname{Im} p_{j}\right)\right| \leq\left|\sin \pi\left(p_{j}+\alpha_{j} s\right)\right| \leq \sqrt{1+\sinh ^{2}\left(\pi \operatorname{Im} p_{j}\right)},
$$

it follows that

$$
\begin{aligned}
\prod_{\operatorname{Re} \alpha_{j}=0} \sqrt{2\left(1+\sinh ^{2}\left(\pi \operatorname{Im} p_{j}\right)\right)} & \geq \prod_{\operatorname{Re} \alpha_{j}=0}\left|\frac{1}{2}\left(1+S\left(\alpha_{j}, s\right)\right)+\left(1-S\left(\alpha_{j}, s\right)\right) \sin \pi\left(p_{j}+\alpha_{j} s\right)\right| \\
& \geq \prod_{\operatorname{Re} \alpha_{j}=0} \min \left\{1,2 \sinh \left(\pi \operatorname{Im} q_{j}\right)\right\}>0,
\end{aligned}
$$

that is, $\prod_{\operatorname{Re} \alpha_{j}=0}\left|(1 / 2)\left(1+S\left(\alpha_{j}, s\right)\right)+\left(1-S\left(\alpha_{j}, s\right)\right) \sin \pi\left(p_{j}+\alpha_{j} s\right)\right| \asymp 1$. Hence,

$$
\frac{\prod_{\operatorname{Re} \beta_{j}=0}\left|(1 / 2)\left(1+S\left(\beta_{j}, s\right)\right)+\left(1-S\left(\beta_{j}, s\right)\right) \sin \pi\left(q_{j}+\beta_{j} s\right)\right|}{\prod_{\operatorname{Re} \alpha_{j}=0}\left|(1 / 2)\left(1+S\left(\alpha_{j}, s\right)\right)+\left(1-S\left(\alpha_{j}, s\right)\right) \sin \pi\left(p_{j}+\alpha_{j} s\right)\right|} \asymp g(t),
$$

where

$$
g(t)=\prod_{\operatorname{Re} \beta_{j}=0}\left|\frac{1}{2}\left(1+S\left(\beta_{j}, i t\right)\right)+\left(1-S\left(\beta_{j}, i t\right)\right) \sin \pi\left(q_{j}-\left(\operatorname{Im} \beta_{j}\right) t\right)\right| .
$$

It, therefore, follows from (3.17) and (3.22) that

$$
\begin{aligned}
& \frac{\prod_{j=1}^{Q}\left|(1 / 2)\left(1+S\left(\beta_{j}, s\right)\right)+\left(1-S\left(\beta_{j}, s\right)\right) \sin \pi\left(q_{j}+\beta_{j} s\right)\right|}{\prod_{j=1}^{P}\left|(1 / 2)\left(1+S\left(\alpha_{j}, s\right)\right)+\left(1-S\left(\alpha_{j}, s\right)\right) \sin \pi\left(p_{j}+\alpha_{j} s\right)\right|} \\
& \asymp g(t) e^{\pi|t|\left(\sum_{\operatorname{Re} \alpha_{j}<0}\left|\operatorname{Re} \alpha_{j}\right|-\sum_{\operatorname{Re} \beta_{j}<0}\left|\operatorname{Re} \beta_{j}\right|\right)} .
\end{aligned}
$$

It is evident that $Y(s) \asymp 1$. Therefore, if $s=i t, t$ is a real number, then Lemma 2.2, formula (3.24), and the fact that $(\operatorname{Im} s) \operatorname{Arg} s=(\pi / 2)|t|$ show that, as $|t| \rightarrow \infty$,

$$
|k(s)| \asymp g(t)|t|^{\Delta_{0}} e^{-\mu t(\ln |t|)} e^{-t M-(\pi / 2)|t| T} e^{\pi|t|\left(\sum_{\operatorname{Re} \alpha_{j}<0}\left|\operatorname{Re} \beta_{j}\right|-\sum_{\operatorname{Re} \beta_{j}<0}\left|\operatorname{Re} \alpha_{j}\right|\right)} .
$$

Since

$$
T+2\left(\sum_{\operatorname{Re} \alpha_{j}<0}\left|\operatorname{Re} \alpha_{j}\right|-\sum_{\operatorname{Re} \beta_{j}<0}\left|\operatorname{Re} \beta_{j}\right|\right)=N,
$$

and since $|t| \asymp(1+|t|)$, as $|t| \rightarrow \infty$, we obtain

$$
|k(s)| \asymp g(t)(1+|t|)^{\Delta_{0}} e^{-\mu t(\ln |t|)} e^{-t M-(\pi / 2)|t| N}, \quad \text { as }|t| \longrightarrow \infty .
$$

Now, if $s=i t, t$ is a real number, and $z \neq 0$, and $\arg z$ is an $\operatorname{argument}$ of $z$, then

$$
\left|k(s) z^{-s}\right|=|k(s)||z|^{-\operatorname{Re} s} e^{(\arg z) \operatorname{Im} s}=|k(s)| e^{t \arg z} .
$$


Therefore, if $z \neq 0$, then

$$
\left|k(s) z^{-s}\right| \asymp g(t)(1+|t|)^{\Delta_{0}} e^{-\mu t(\ln |t|)-t(M-\arg z)-(\pi / 2)|t| N} \quad \text { as }|t| \longrightarrow \infty .
$$

Applying Lemma 3.1 gives the conclusion of Theorem 3.2.

REMARK 3.3. If $|M| \leq(\pi / 2) N$ and the integral $\int_{\Omega_{c}} k(s) z^{-s} d s$ converges absolutely for some, real or complex, number $z_{0}$, then it converges absolutely for all positive real $z$. For if either of the conditions (1) or (2) of Theorem 3.2 is satisfied for some $z_{0}$, then it is satisfied for any $z$ with $\arg z=0$ (the point $z=0$ is tacitly excluded).

If $|M|>(\pi / 2) N$, then the integral $\int_{\Omega_{c}} k(s) z^{-s} d s$ may converge for some complex $z_{0}$, but diverges for all real $z$. This is a striking difference with the Mellin-Barnes integral with real $(\alpha)_{P}$ and $(\beta)_{Q}$, where the convergence domain always contains some real number.

Because $\Lambda_{c}$ is a special case of the contour $\Omega_{c}$ we have the following theorem.

THEOREM 3.4. The H-function (1.4) exists if

(1) $\mu=0, N>0$, and for $|M-\arg z|<(\pi / 2) N$, or

(2) $\mu=0, N \geq 0, \arg z=M \pm(\pi / 2) N$, and $\Delta_{c}=\Delta_{0}+c T<-1$.

REMARK 3.5. A close look at the asymptotics of $k(s)$ shows that the $H$-function (1.4) exists only if $\mu=0$. Hence, in general, the $H$-function is not analytic with respect to the parameters $\alpha_{j}, j=1, \ldots, P$, and $\beta_{j}, j=1, \ldots, Q$, although it is analytic with respect to the parameters $p_{j}, j=1, \ldots, P$, and $q_{j}, j=1, \ldots, Q$.

REMARK 3.6. If $N>0$, then the $H$-function is analytic in the sector $|M-\arg z|<$ $(\pi / 2) N$.

\section{Properties}

4.1. Power multiplier. We have

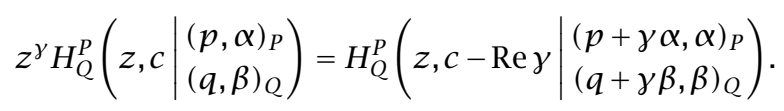

The change of variables $s \rightarrow s-\gamma$ gives

$$
\begin{aligned}
z^{\gamma} H_{Q}^{P}\left(z, c \mid \begin{array}{l}
(p, \alpha)_{P} \\
(q, \beta)_{Q}
\end{array}\right) & =\frac{1}{2 \pi i} \int_{\Lambda_{c}} k(s) z^{-s+\gamma} d s=\frac{1}{2 \pi i} \int_{\Lambda_{c-\operatorname{Re}(\gamma)}} k(s+\gamma) z^{-s} d s \\
& =H_{Q}^{P}\left(z, c-\operatorname{Re} \gamma \mid \begin{array}{l}
(p+\gamma \alpha, \alpha)_{P} \\
(q+\gamma \beta, \beta)_{Q}
\end{array}\right) .
\end{aligned}
$$

4.2. Reflection property. We have

$$
H_{Q}^{P}\left(\frac{1}{z}, c \mid \begin{array}{c}
(p, \alpha)_{P} \\
(q, \beta)_{Q}
\end{array}\right)=H_{Q}^{P}\left(z,-c \mid \begin{array}{c}
(p,-\alpha)_{P} \\
(q,-\beta)_{Q}
\end{array}\right) .
$$


This follows easily from the fact that

$$
\frac{1}{2 \pi i} \int_{\Lambda_{c}} k(s) z^{s} d s=\frac{1}{2 \pi i} \int_{\Lambda_{-c}} k(-s) z^{-s} d s
$$

4.3. The Mellin transform. Let $c$ be a real number such that:

(a) $\operatorname{Re}\left(-p_{j} / \alpha_{j}\right)>c$, whenever $\operatorname{Re} \alpha_{j}<0$, and $\operatorname{Re}\left(-p_{j} / \alpha_{j}\right)<c$, whenever $\operatorname{Re} \alpha_{j}>0$.

If $|M| \leq(\pi / 2) N$, and the parameters and variable $z$ satisfy the conditions of Theorem 3.2, then

$$
\int_{0}^{\infty} H_{Q}^{P}\left(x, c \mid \begin{array}{c}
(p, \alpha)_{P} \\
(q, \beta)_{Q}
\end{array}\right) x^{s-1} d x=\frac{\prod_{j=1}^{P} \Gamma\left(p_{j}+\alpha_{j} s\right)}{\prod_{j=1}^{Q} \Gamma\left(q_{j}+\beta_{j} s\right)} .
$$

Indeed, condition (a) guarantees that the contour $\Lambda_{c}$ in formula (1.4) can be taken as a vertical line. Therefore, $H_{Q}^{P}\left(x, c \mid \begin{array}{c}(p, \alpha)_{P} \\ (q, \beta)_{Q}\end{array}\right)$ is the inverse Mellin transform of the function $k(s)$ along $\Lambda_{c}$. Since $k(s)$ is differentiable on $\Lambda_{c}$ and by the conditions of Theorem 3.2, $k(s) \in L^{1}\left(\Lambda_{c}\right)$, it follows from [17] that $k(s)$ is the Mellin transform of $H_{Q}^{P}\left(x, c \mid \begin{array}{l}(p, \alpha)_{P} \\ (q, \beta)_{Q}\end{array}\right)$.

5. Examples. (a) It is clear from the definition that the Meijer $G$-function and Fox $H$-function are special cases of the $H$-function with complex parameters (1.4).

(b) In the formula 6.421 .2 of [6],

$$
\int_{-\infty}^{\infty} \frac{e^{\pi i t x}}{\Gamma(a+x) \Gamma(b-x) \Gamma(c+\beta x) \Gamma(d-\beta x)} d x=0, \quad \operatorname{Re}(a+b+c+d)>2,|t|>|\beta|+1,
$$

and $t, \beta$ are real; put $x=-i s$, then

$$
\begin{aligned}
\int_{-\infty}^{\infty} \frac{e^{\pi i t x}}{\Gamma(a+x) \Gamma(b-x) \Gamma(c+\beta x) \Gamma(d-\beta x)} d x & \\
=-i \int_{-i \infty}^{i \infty} \frac{e^{\pi t s}}{\Gamma(a-i s) \Gamma(b+i s) \Gamma(c-i \beta s) \Gamma(d+i \beta s)} & d s .
\end{aligned}
$$

Thus,

$$
H_{4}^{0}\left(e^{-\pi t},\left.0\right|_{(a,-i),(b, i),(c,-i \beta),(d, i \beta)}\right)=0, \quad \operatorname{Re}(a+b+c+d)>2,|t|>|\beta|+1,
$$

and $t, \beta$ are real.

Note that this is a particular value of an $H$-function with complex parameters which is not a special case of the classical Fox $H$-function.

(c) Using formulae 6.414.1-6.414.6, 6.421.1, 6.421.3, and 6.421.4 of [6] along with 
the substitution $x=-i$, we get particular values of $H$-functions with complex parameters:

$$
\begin{aligned}
& H_{1}^{1}\left(1,0 \mid \begin{array}{c}
(a,-i) \\
(b, i)
\end{array}\right)=0, \quad \operatorname{Im} a \neq 0, \operatorname{Re}(a-b)<-1, \\
& H_{2}^{0}(1,0 \mid(a,-i),(b, i))=\frac{2^{a+b-3}}{\pi \Gamma(a+b-1)}, \quad \operatorname{Re}(a+b)>1, \\
& H_{2}^{2}\left(1,0 \mid \begin{array}{l}
(a,-i),(b,-i) \\
(c,-i),(d,-i)
\end{array}\right)=0, \operatorname{Re}(c+d-a-b)>1 ; \operatorname{Im} a, \operatorname{Im} b>0, \\
& H_{2}^{2}\left(1,0 \mid \begin{array}{l}
(a,-i),(b,-i) \\
(c,-i),(d,-i)
\end{array}\right)=\frac{\pi[\operatorname{signIm}(c-d)] \Gamma(c+d-a-b)}{[\sin \pi(a-b)] \Gamma(c-a) \Gamma(c-b) \Gamma(d-a) \Gamma(d-b)}, \\
& \operatorname{Re}(c+d-a-b)>1 ; \operatorname{Im} a, \operatorname{Im} b<0, \\
& H_{3}^{1}\left(\begin{array}{l|c}
(a-b-c+1,-i) \\
(a,-i),(b, i),(c,-i)
\end{array}\right)=\frac{\exp [(\pi(f-c) i / 2) \operatorname{signIm} f]}{2 \Gamma(b+c-1) \Gamma((a+b) / 2) \Gamma((c-f+1) / 2)}, \\
& \operatorname{Re}(b+c)>1, f=a-b-c+1, \operatorname{Im} f \neq 0, \\
& H_{4}^{0}(1,0 \mid(a,-i),(b, i),(c,-i),(d, i))=\frac{(1 / 2 \pi) \Gamma(a+b+c+d-3)}{\Gamma(a+b-1) \Gamma(b+c-1) \Gamma(c+d-1) \Gamma(d+a-1)}, \\
& \operatorname{Re}(a+b+c+d)>3 \\
& H_{0}^{2}\left(e^{-2(\pi n+\theta)}, 0 \mid(a,-i),(b, i)\right)=\Gamma(a+b)(2 \cos \theta)^{a-b}(\exp ((b-a) i \theta)) \\
& \times\left(\eta_{n}(b) \exp (2 n \pi b i)-\eta_{n}(-a) \exp (-2 n \pi a i)\right), \\
& \operatorname{Re}(a+b)<1 ;-\frac{\pi}{2}<\theta<\frac{\pi}{2} ; \\
& \eta_{n}(\zeta)=\frac{1}{2} \operatorname{sign}\left(\frac{1}{2}-n\right)\left(1-\operatorname{sign}\left[\left(\frac{1}{2}-n\right) \operatorname{Im} \zeta\right]\right),
\end{aligned}
$$

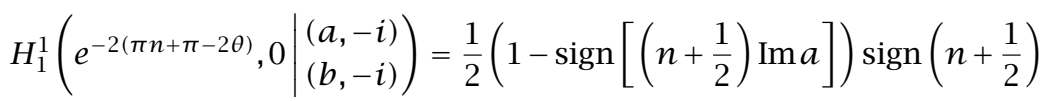

$$
\begin{aligned}
& \times \frac{(2 \cos \theta)^{b-a-1}}{\Gamma(b-a)} \exp (-2 \pi n+\pi-\theta) a i+\theta i(b-1), \\
& \operatorname{Re}(b-a)>0,-\frac{\pi}{2}<\theta<\frac{\pi}{2}, n \text { is an integer. }
\end{aligned}
$$

We remark that none of the above $H$-functions is a special case of the classical Fox $H$-function.

6. $H$-function with complex parameters as a Fourier kernel. Let the conditions of Theorem 3.2 be fulfilled, and moreover,

$$
M=N=0, \quad \Delta_{1 / 2}=-2 .
$$


Assume further that

$$
\begin{aligned}
& \operatorname{Re}\left(\frac{p_{j}}{\alpha_{j}}\right)>-\frac{1}{2}, \quad \text { if } \operatorname{Re} \alpha_{j}>0, \\
& \operatorname{Re}\left(\frac{p_{j}}{\alpha_{j}}\right)<-\frac{1}{2}, \quad \text { if } \operatorname{Re} \alpha_{j}<0, \\
& \operatorname{Re}\left(\frac{p_{j}}{\alpha_{j}}\right) \neq-\frac{1}{2}, \quad \text { if } \operatorname{Re} \alpha_{j}=0 .
\end{aligned}
$$

Conditions (6.2) guarantee that the vertical line $((1 / 2)-i \infty,(1 / 2)+i \infty)$ can be taken as the contour $\Lambda_{1 / 2}$ of integration in the definition of $H_{Q}^{P}\left(z,(1 / 2) \mid \begin{array}{c}(p, \alpha)_{p} \\ (q, \beta)_{Q}\end{array}\right)$.

THEOREM 6.1. The H-integral transform

$$
g(x)=x^{2} \frac{d^{2}}{d x^{2}} \int_{0}^{\infty} H_{Q}^{P}\left(x y, \frac{1}{2} \mid \begin{array}{c}
(p, \alpha)_{P} \\
(q, \beta)_{Q}
\end{array}\right) f(y) d y
$$

is a bounded integral transform from $L^{2}\left(\mathbb{R}_{+}\right)$onto itself, and the inverse transform has the form

$$
f(x)=x^{2} \frac{d^{2}}{d x^{2}} \int_{0}^{\infty} H_{P+2}^{Q+2}\left(x y, \frac{1}{2} \mid \begin{array}{l}
(q+\beta,-\beta)_{Q},(1,-1),(0,1) \\
(p+\alpha,-\alpha)_{P},(3,-1),(2,1)
\end{array}\right) g(y) d y .
$$

Proof. Due to the assumptions (6.1), $|k(s)| \asymp 1 /|s|^{2}$, and therefore, $k(s) \in L_{2}$ $((1 / 2)-i \infty,(1 / 2)+i \infty)$. Hence, $H_{Q}^{P}\left(x,(1 / 2) \mid \begin{array}{c}(p, \alpha)_{p} \\ (q, \beta)_{Q}\end{array}\right)$ exists for $x>0$ and belongs to $L^{2}\left(\mathbb{R}_{+}\right)$, since it is the inverse Mellin transform of $k(s)$ [17]. Consequently, the integral (6.3) converges absolutely if $f \in L^{2}\left(\mathbb{R}_{+}\right)$. By the Mellin convolution theorem [17], we obtain

$$
g(x)=\frac{x^{2}}{2 \pi i} \frac{d^{2}}{d x^{2}} \int_{(1 / 2)-i \infty}^{(1 / 2)+i \infty} k(s) f^{*}(1-s) x^{-s} d s
$$

where $f^{*}$ is the Mellin transform of $f$.

Due to the assumption on $k(s)$, and the fact that $f^{*}(1-s) \in L_{2}((1 / 2)-i \infty$, $(1 / 2)+i \infty)$, it follows that $s(s-1) k(s) f^{*}(1-s) \in L_{2}((1 / 2)-i \infty,(1 / 2)+i \infty)$. Hence,

$$
g(x)=\frac{1}{2 \pi i} \int_{(1 / 2)-i \infty}^{(1 / 2)+i \infty} s(s+1) k(s) f^{*}(1-s) x^{-s} d s
$$

where the integral is understood in the sense of $L_{2}((1 / 2)-i \infty,(1 / 2)+i \infty)$. Therefore,

$$
g^{*}(s)=s(s+1) k(s) f^{*}(1-s)
$$

or

$$
f^{*}(s)=\frac{g^{*}(1-s)}{(1-s)(2-s) k^{*}(1-s)}=\frac{g^{*}(1-s)}{(1-s)(2-s)} \frac{\prod_{j=1}^{Q} \Gamma\left(q_{j}+\beta_{j}-\beta_{j} s\right)}{\prod_{j=1}^{P} \Gamma\left(p_{j}+\alpha_{j}-\alpha_{j} s\right)}
$$


Consequently,

$$
\begin{aligned}
f(x) & =\frac{1}{2 \pi i} \int_{(1 / 2)-i \infty}^{(1 / 2)+i \infty} \frac{\Gamma(1-s) \Gamma(s)}{\Gamma(3-s) \Gamma(2+s)} \frac{\prod_{j=1}^{Q} \Gamma\left(q_{j}+\beta_{j}-\beta_{j} s\right)}{\prod_{j=1}^{P} \Gamma\left(p_{j}+\alpha_{j}-\alpha_{j} s\right)} s(s+1) g^{*}(1-s) x^{-s} d s \\
& =\frac{x^{2}}{2 \pi i} \frac{d^{2}}{d x^{2}} \int_{(1 / 2)-i \infty}^{(1 / 2)+i \infty} \frac{\Gamma(1-s) \Gamma(s)}{\Gamma(3-s) \Gamma(2+s)} \frac{\prod_{j=1}^{Q} \Gamma\left(q_{j}+\beta_{j}-\beta_{j} s\right)}{\prod_{j=1}^{P} \Gamma\left(p_{j}+\alpha_{j}-\alpha_{j} s\right)} g^{*}(1-s) x^{-s} d s \\
& =x^{2} \frac{d^{2}}{d x^{2}} \int_{0}^{\infty} H_{P+2}^{Q+2}\left(x y, \frac{1}{2} \mid \begin{array}{l}
(q+\beta,-\beta)_{Q},(1,-1),(0,1) \\
(p+\alpha,-\alpha)_{P},(3,-1),(2,1)
\end{array}\right) g(y) d y .
\end{aligned}
$$

This completes the proof of the theorem.

ACKNOWLEDGEMENTS. The authors wish to thank Professor H. M. Srivastava for the helpful comments. Fadhel A. Al-Musallam was partially supported by Kuwait University Research Administration under grant SM 167.

\section{REFERENCES}

[1] W. N. Bailey, Generalized Hypergeometric Series, Cambridge Tracts in Mathematics and Mathematical Physics, no. 32, Stechert-Hafner, New York, 1964. MR 32\#2625.

[2] E. W. Barnes, A new development of the theory of hypergeometric functions, Proc. London Math. Soc. 6 (1907), 141-177.

[3] A. L. Dixon and W. L. Ferrar, A class of discontinuous integrals, Quart. J. Math. Oxford Ser. 7 (1936), 81-96. Zbl 014.10804.

[4] A. Erdélyi, W. Magnus, F. Oberhettinger, and F. G. Tricomi, Higher Transcendental Functions. Vol. I, McGraw-Hill Book Co., New York, 1953, Bateman Manuscript Project. Zbl 051.30303.

[5] C. Fox, The $G$ and $H$ functions as symmetrical Fourier kernels, Trans. Amer. Math. Soc. 98 (1961), 395-429. MR 24\#A1427. Zbl 096.30804.

[6] I. S. Gradshteyn and I. M. Ryzhik, Table of Integrals, Series, and Products, Academic Press [Harcourt Brace Jovanovich Publishers], Ontario, 1980, Corrected and enlarged edition edited by Alan Jeffrey. Incorporating the fourth edition edited by Yu. V. Geronimus and M. Yu. Tseytlin; translated from Russian. MR 81g:33001. Zbl 521.33001.

[7] A. M. Mathai, Distributions of Test Statistics: Exact \& Asymptotic, Null \& Non-null, Methods/Comparisons/Research Frontiers, The American Sciences Press Series in Mathematical and Management Sciences, vol. 18, American Sciences Press, Ohio, 1989, Reprinted from “Amer. J. Math. Management Sci. 9 (1989), no. 1-2”. Zbl 743.62017.

[8] A. M. Mathai and H. J. Haubold, Modern Problems in Nuclear and Neutrion Astrophysics, Akademie-Verlag, Berlin, 1988.

[9] A. M. Mathai and R. K. Saxena, Generalized Hypergeometric Functions with Applications in Statistics and Physical Sciences, vol. 348, Springer-Verlag, New York, 1973, Lecture Notes in Mathematics. MR 57\#3471. Zbl 272.33001.

[10] _ The H-Function with Applications in Statistics and other Disciplines, Halsted Press [John Wiley \& Sons], London, 1978. MR 80a:33007. Zbl 382.33001.

[11] C. S. Meijer, On the G-function. I-VIII, Nederl. Akad. Wetensch. Proc. Ser. A 49 (1946), 227-237, 344-356, 457-469, 632-641, 765-772, 936-943, 1063-1072, 1165-1175 (1946); Indag. Math. 8, 124-134, 213-225, 312-324, 391-400, 468-475, 595-602, 661-670, 713-723 (1946). Zbl 060.19901.

[12] H. J. Mellin, Abrip einer einhaitlichen Theorie der Gamma und der hypergeometrischen Funktionen, Math. Ann. 68 (1910), 305-337 (German). 
[13] S. Pincherle, Sulle funzioni ipergeometriche generalizzate, Atti Accad. Naz. Lincei Rend. 4 (1888), no. 4, 694-700, 792-800 (Italian).

[14] A. P. Prudnikov, Y. A. Brychkov, and O. I. Marichev, Integrals and Series, "Nauka", Moscow, 1986 (Russian), Dopolnitelnye glavy. [Supplementary chapters]. MR 88f:00012. Zbl 606.33001.

[15] L. J. Slater, Generalized Hypergeometric Functions, Cambridge University Press, Cambridge, 1966. MR 34\#1570. Zbl 135.28101.

[16] H. M. Srivastava, K. C. Gupta, and S. P. Goyal, The H-functions of One and Two Variables, South Asian Publishers Pvt. Ltd., New Delhi, 1982, with applications. MR 84f:33011. Zbl 506.33007.

[17] E. C. Tichmarsch, An Introduction to the Theory of Fourier Integrals, Oxford University Press, Oxford, 1948.

FADHEL A. AL-Musallam: Department of MATHEMATICS AND Computer SCIENCE, KuWAit UNIVERSITY, P.O. BOX 5969 SAFAT, 13060, KUWAIT

E-mail address: musa11 am@mcs. sci . kuniv. edu. kw

Vu Kim TuAN: Department of Mathematics AND COMPUTER SCIENCE, KuWAit UNIVERSity, P.O. BOX 5969 SAFAT, 13060, KUWAIT

E-mail address: vu@mcs . sci . kuniv . edu . kw 


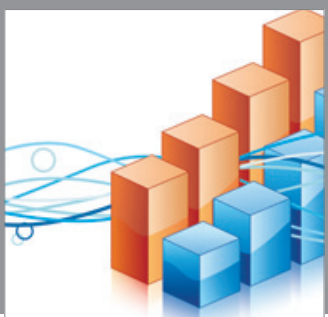

Advances in

Operations Research

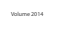

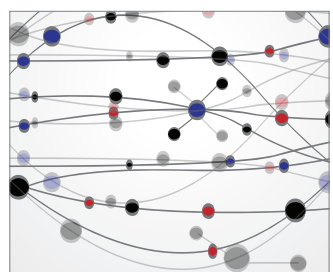

\section{The Scientific} World Journal
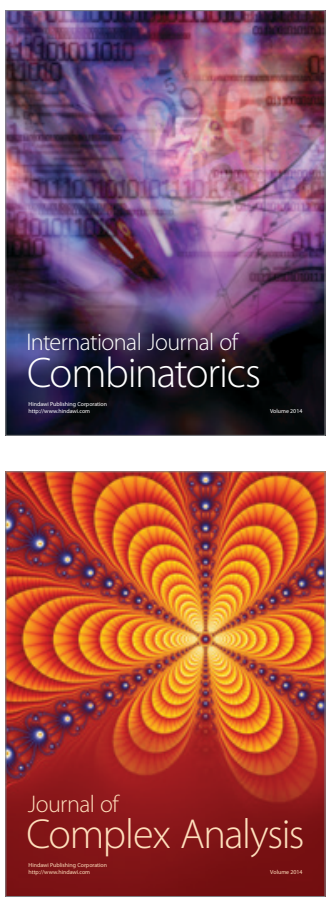

International Journal of

Mathematics and

Mathematical

Sciences
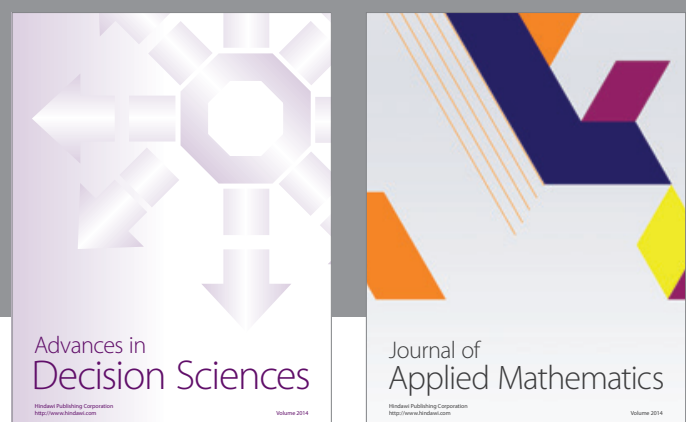

Journal of

Applied Mathematics
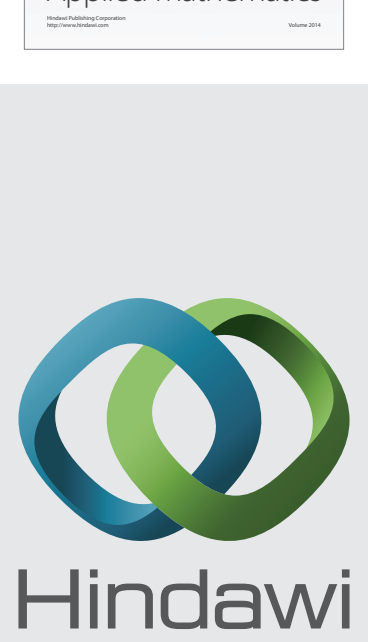

Submit your manuscripts at http://www.hindawi.com
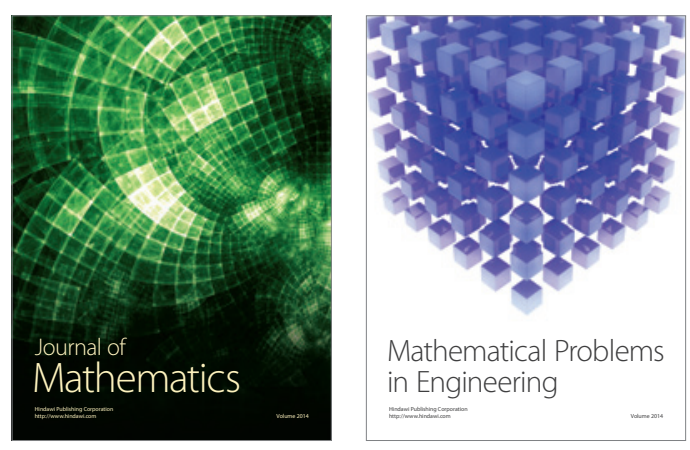

Mathematical Problems in Engineering
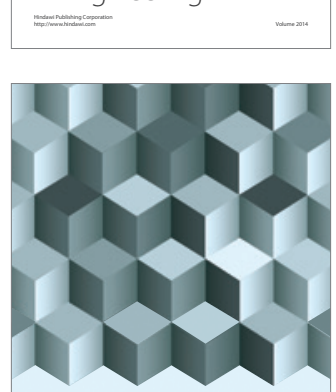

Journal of

Function Spaces
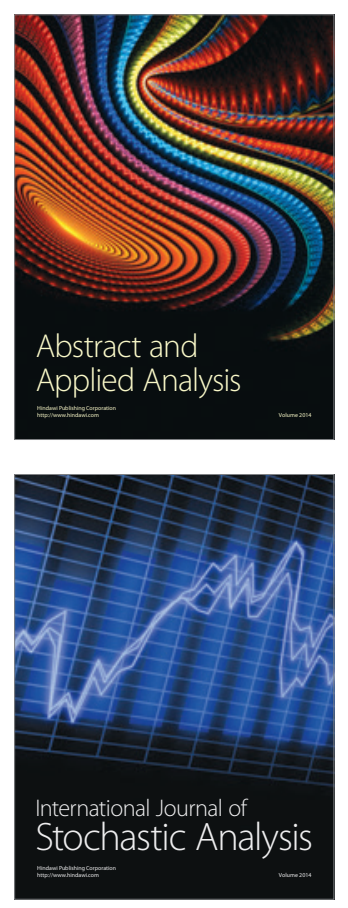

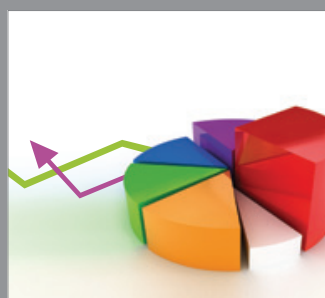

ournal of

Probability and Statistics

Promensencen
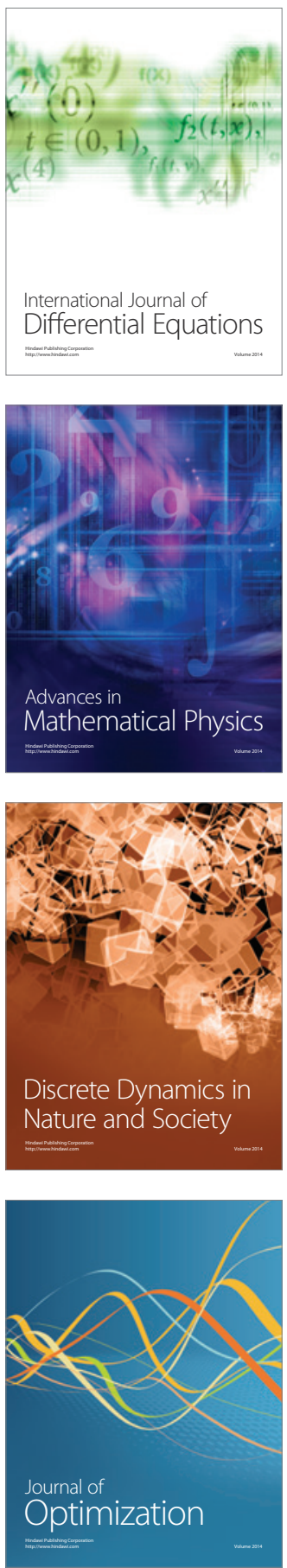